\title{
Isospectral Domains in Euclidean 3-Space
}

\author{
Christopher Cox ${ }^{* *}$ \\ Department of Mathematics \\ lowa State University \\ Ames, lowa 50011 USA
}

Received: November 13, 2011

Accepted: February 8, 2012

\section{ABSTRACT}

The question as to whether the shape of a drum can be heard has existed for around fifty years. The simple answer is 'no' as shown through the construction of isospectral domains. Isospectral domains are non-isometric domains that display the same spectra of frequencies of sound. These frequencies, deduced from the eigenvalues of the Laplacian, are determined by solving the wave equation in a domain $\Omega$, where $\partial \Omega$ is subject to Dirichlet boundary conditions. This paper presents methods to expand the already existing two dimensional transplantation proof into Euclidean 3space and, through these means, provides a number of three dimensional isospectral domains.

\section{INTRODUCTION}

In 1966, Mark Kac proposed the question, "Can one hear the shape of a drum?" [see 6] This question refers to the eigenvalues of a vibrating membrane. These eigenvalues arise from the solution of a wave equation on a region $\Omega$ bounded with Dirichlet boundary conditions. In other words,

$$
\begin{gathered}
\nabla^{2} \varphi(x, y, t)=\frac{1}{k^{2}} \frac{\partial^{2} \varphi(x, y, t)}{\partial t^{2}}, \quad(x, y) \in \Omega \\
\varphi(x, y, t)=0, \quad(x, y) \in \partial \Omega
\end{gathered}
$$

where, in this case, $\nabla^{2} \varphi=\left(\frac{\partial^{2}}{\partial x^{2}}+\frac{\partial^{2}}{\partial y^{2}}\right) \varphi$ and $k^{2}$ is determined from the density and tension of the membrane. A procedure for solving this second order partial differential equation for $\varphi$, assuming Cartesian symmetry, is the separation of variables wherein $\varphi(x, y, t)=\psi(x, y) T(t)$. By utilizing this substitution, we obtain a second order ordinary differential equation and a Helmholtz equation:

$$
\begin{gathered}
T^{\prime \prime}(t)+k^{2} \lambda T(t)=0 \\
\nabla^{2} \psi(x, y)+\lambda \psi(x, y)=0
\end{gathered}
$$

**Email: cocox@iastate.edu
By applying the substitution $\psi(x, y)=$ $X(x) Y(y)$, we obtain two more second order ordinary differential equations:

$$
\begin{aligned}
& X^{\prime \prime}(x)+\alpha X(x)=0 \\
& Y^{\prime \prime}(y)+\beta Y(y)=0
\end{aligned}
$$

where $\lambda=\alpha+\beta$. Solutions of these three differential equations yield

$$
\begin{aligned}
& T(t)=A \sin (k \sqrt{\lambda} t)+B \cos (k \sqrt{\lambda} t) \\
& X(x)=C \sin (\sqrt{\alpha} x)+D \cos (\sqrt{\alpha} x) \\
& Y(y)=E \sin (\sqrt{\beta} y)+F \cos (\sqrt{\beta} y)
\end{aligned}
$$

The eigenvalues of this wave equation are $\lambda, \alpha$, and $\beta$, which depend on the region $\Omega$. If $\Omega$ were to be a rectangular region of side lengths $L$ and $H$, the eigenvalues would be $\alpha=\left(\frac{n \pi}{L}\right)^{2}, \beta=\left(\frac{m \pi}{H}\right)^{2}$, and $\lambda=\alpha+\beta$, where $n, m \in \mathbb{N}$. Also, due to the boundary conditions, $D$ and $F$ go to zero. By using the fact that $\varphi(x, y, t)=$ $T(t) X(x) Y(y)$ and the concept of linear superposition, the final solution of the wave equation is as follows: 

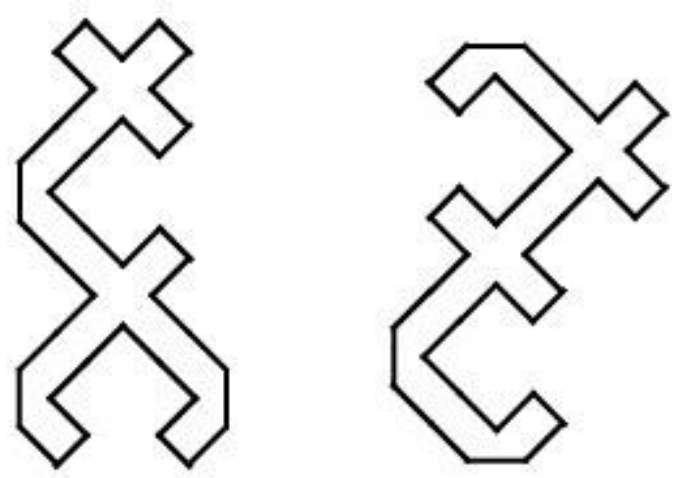

Figure 1. These two domains will produce identical spectra of frequencies when played. They can be simplified into two heptaboloes of similar orientations.

$$
\begin{aligned}
\varphi(x, y, t)=\sum_{n=1}^{\infty} \sum_{m=1}^{\infty}\left[A_{n m} \sin \left(t k \sqrt{\left(\frac{n \pi}{L}\right)^{2}+\left(\frac{m \pi}{H}\right)^{2}}\right)\right. \\
\left.+B_{n m} \cos \left(t k \sqrt{\left(\frac{n \pi}{L}\right)^{2}+\left(\frac{m \pi}{H}\right)^{2}}\right)\right]\left[\sin \left(\frac{n \pi x}{L}\right) \sin \left(\frac{m \pi y}{H}\right)\right]
\end{aligned}
$$

The coefficients $A_{n m}$ and $B_{n m}$ are determined by the initial conditions of the deformation of the membrane.

In relation to this information, Kac wondered if two non-isometric domains, $\Omega_{1}$ and $\Omega_{2}$, could produce the same spectra of frequencies, that is, have the same eigenvalues. The question remained unanswered until 1992 when Gordon et al. [see 5] presented two domains that had different shapes but produced identical spectra [figure 1].

Here we provide methods for the creation of isospectral domains in Euclidean 3-space by extending the 2-space transplantation methods to incorporate the added dimension. In section 2, we shall extend Dirichlet boundary conditions and folding rules to 3 -space. In section 3 , we shall present three dimensional isospectral domains with explanations of transplantation methods. Finally, further areas in which to extend research and applications will be presented.

\section{PRELIMINARIES}

In order to understand the transplantation proofs of isospectrality in 3- space, one must first understand how to extend Dirichlet boundary conditions to higher dimensions along with the extensions of basic folding rules.

\section{a. Boundary conditions}

A wave equation, $\varphi$, on a given two dimensional region, $\Omega$, with Dirichlet boundary conditions, $\left.\varphi\right|_{\partial \Omega}=0$, describes the movement of a drum: e.g. a two dimensional membrane bounded on a one dimensional boundary where $\varphi$ yields a three dimensional coordinate. By extension, if $\Omega \in \mathbb{R}^{n}$, then $\partial \Omega \in \mathbb{R}^{n-1}$ and $\varphi \in \mathbb{R}^{n+1}$. In the case of $\Omega \in \mathbb{R}^{3}$, the wave equation will yield a four dimensional coordinate while the Dirichlet conditions require the domain to be clamped by a two dimensional boundary. A wave equation of a domain of this form will, in fact, describe a three dimensional drum vibrating into the fourth dimension. This allows construction of the regions in our space; however, visualization of the vibrations is impossible given our restricted directions of movement.

When solving this new wave equation, $\varphi$ must take the form of $\varphi(x, y, z, t)$ due to the extra parameter. A sample 

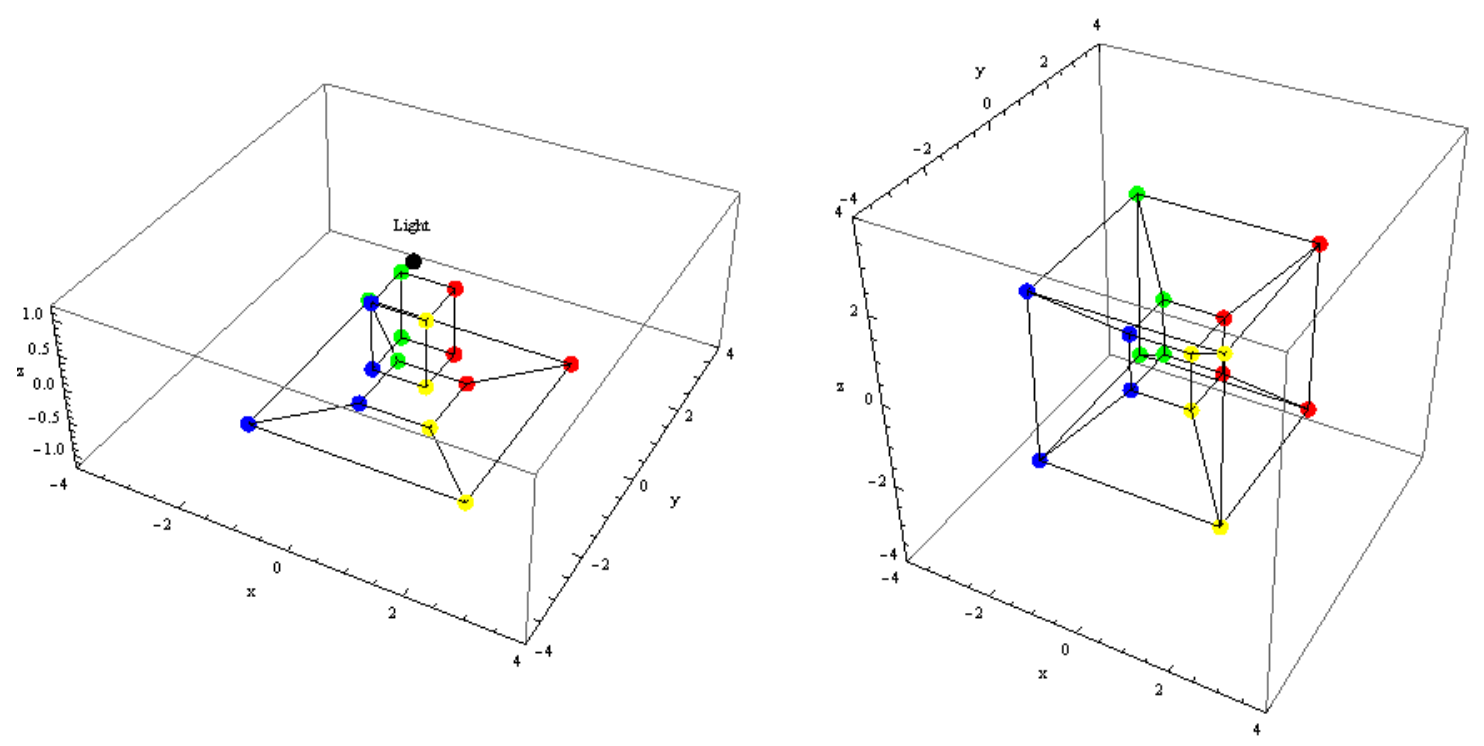

Figure 2. A light source in $n$-space casts a shadow of an $n$-cube into (n-1)-space. Note that the closer a line/face/volume is to the light source, the larger it appears in the projection and vice versa. This fact allows a view of higher dimensional folding via projective geometry. The colors of the vertices are to show the connections between the figure and its projective image.

solution to a wave equation in three dimensions with Dirichlet boundary conditions would be that of a vibrating rectangular prism of side lengths $L, H$, and $D$ respectively. Similar to the earlier problem of the vibrating rectangular membrane, the solution of this wave equation would be as follows:

$$
\begin{aligned}
\varphi(x, y, z, t)=\sum_{n=1}^{\infty} & \sum_{m=1}^{\infty} \sum_{p=1}^{\infty}\left[A_{n m p} \sin \left(t k \sqrt{\left(\frac{n \pi}{L}\right)^{2}+\left(\frac{m \pi}{H}\right)^{2}+\left(\frac{p \pi}{D}\right)^{2}}\right)\right. \\
& \left.+B_{n m p} \cos \left(t k \sqrt{\left(\frac{n \pi}{L}\right)^{2}+\left(\frac{m \pi}{H}\right)^{2}+\left(\frac{p \pi}{D}\right)^{2}}\right)\right]\left[\sin \left(\frac{n \pi x}{L}\right) \sin \left(\frac{m \pi y}{H}\right) \sin \left(\frac{p \pi z}{D}\right)\right]
\end{aligned}
$$

where $n, m, p \in \mathbb{N}$, and, as in the earlier problem, $A_{n m p}$ and $B_{n m p}$ are determined through the initial conditions of deformation.

\section{b. Folding rules}

The best way in which to visualize folding in higher dimensions is through what is known as projective geometry. Projective geometry is a method that can be used to embed an $n$-dimensional object into $(n-1)$ space. This method works by considering a hypothetical light source in $n$-space casting a shadow of an object onto ( $n-1)$-space. The simplest case is that of $n=3$ because of our experiences, but $n=4$ becomes more challenging. In order to visualize this case, one must consider a light source in 4-space acting upon an object to cast a three dimensional 'shadow.' As an example, consider the case of a three dimensional cube and a four dimensional hypercube being projected onto the next lower dimension.

Consider a region, $\Omega \in \mathbb{R}^{n}$, divided into two smaller isometric regions, 

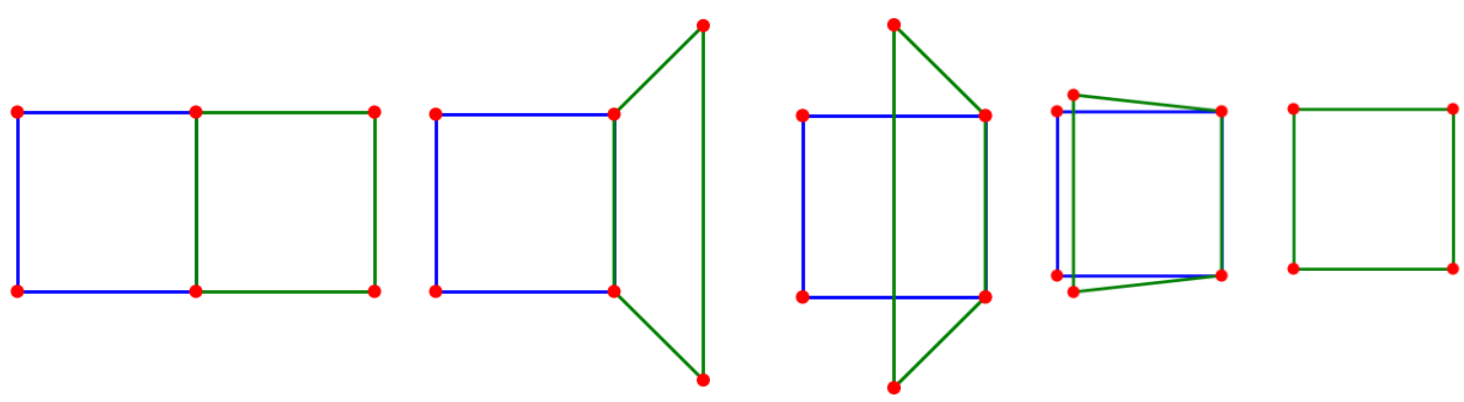

Figure 3a. The blue box denotes $\omega_{1}$ while green denotes $\omega_{2}$. The line connecting the two regions is $\partial \Omega_{I}$. In the third step, $\omega_{2}$ becomes $-\omega_{2}$. The final step shows $-\omega_{2}$ overlapping $\omega_{1}$.
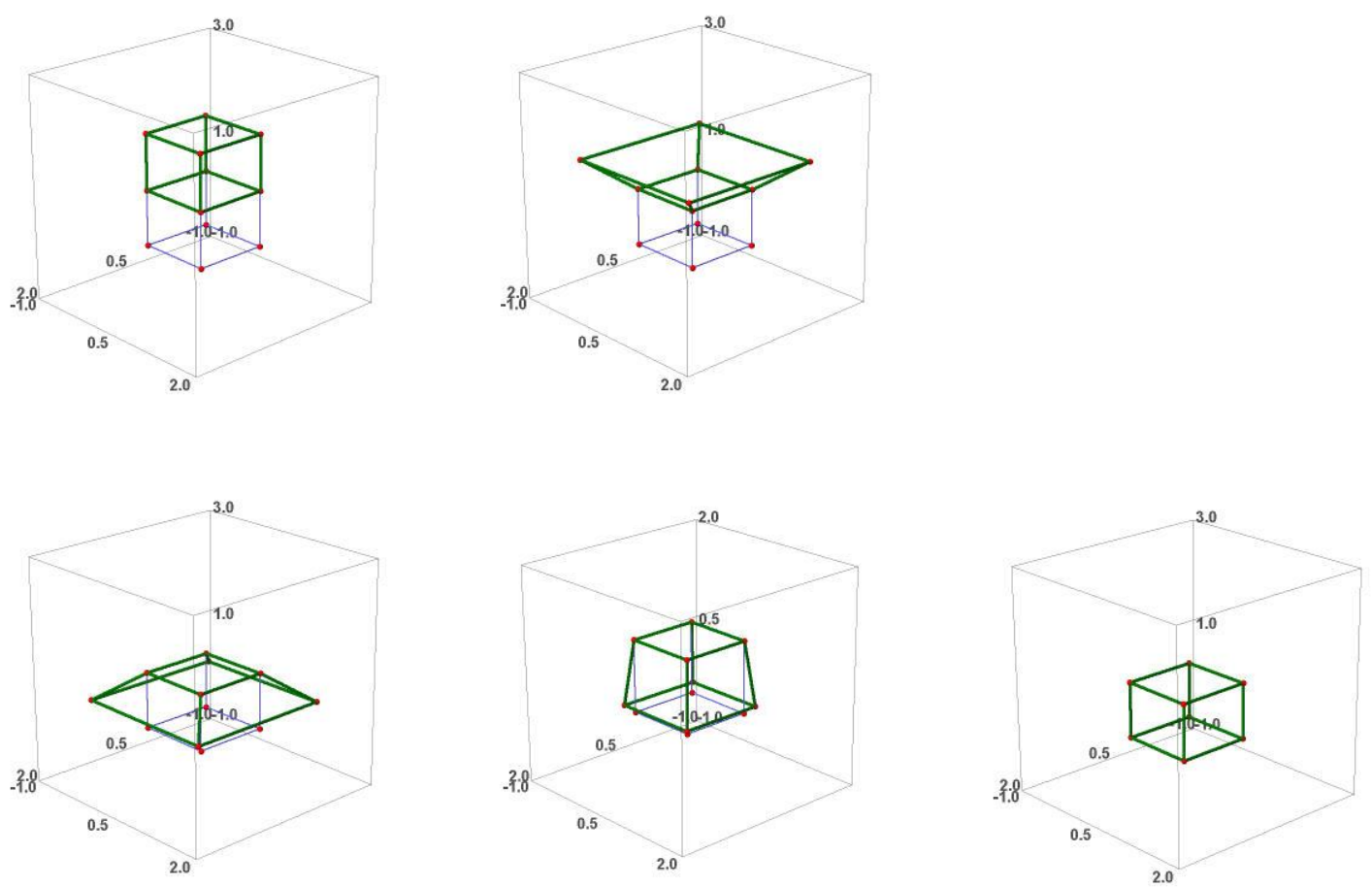

Figure $\mathbf{3 b}$. This is simply an extension of figure $3 \mathrm{a}$ into the next higher dimension where $\omega_{1}$ and $\omega_{2}$ are volumes instead of areas and $\partial \Omega_{I}$ is a face.

$\Omega=\omega_{1} \cup \omega_{2}$. These two smaller domains are connected by their common boundary $\partial \Omega_{I}=\partial \omega_{1} \cap \partial \omega_{2}$. In order to fold $\omega_{2}$ onto $\omega_{1}$ across $\partial \Omega_{I}$, one must cross through $\mathbb{R}^{n+1}$. To best visualize this, take a piece of paper and cut out a region, $\Omega$. Divide this region in half with a line, $\partial \Omega_{I}$, such that the two halves, $\omega_{1}$ and $\omega_{2}$, are reflections of each other about $\partial \Omega_{I}$. Label these two halves as such and the back side of them as $-\omega_{1}$ and $-\omega_{2}$ respectively. Now fold $\omega_{2}$ onto $\omega_{1}$ across $\partial \Omega_{I}$. Notice that the paper must cross into the third dimension in order to complete the fold and that, not only is $\omega_{2}$ reflected onto $\omega_{1}$, it is reversed as well so that $-\omega_{2}$ overlaps $\omega_{1}$. 
$\Omega_{1}$

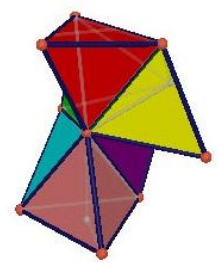

$\Omega_{2}$

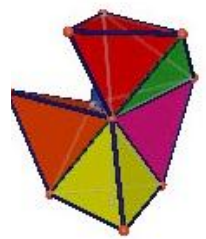

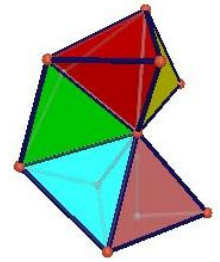
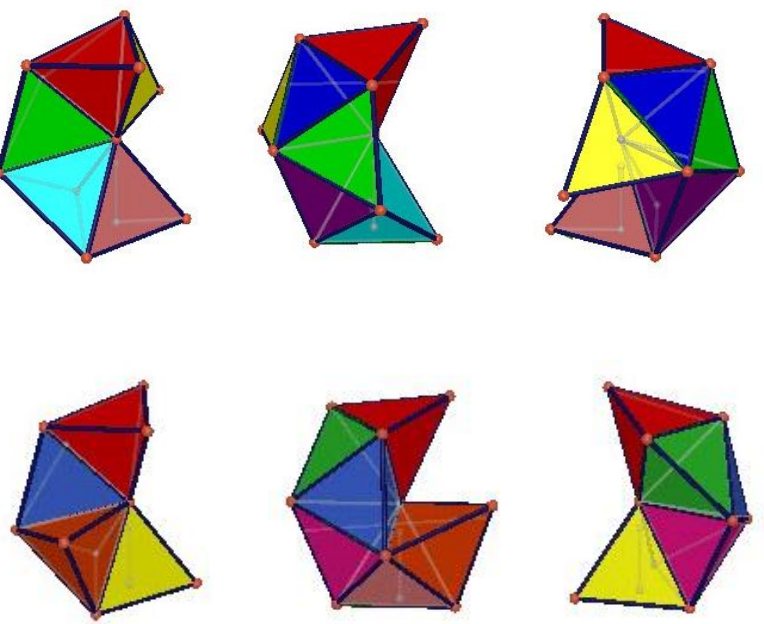

Figure 4. Four different views are shown of $\Omega_{1}$ and $\Omega_{2}$ from different angles. The colors are for the eye and carry no other significance.

Now take this same case by using projective geometry to visualize the fold. Instead of seeing the actual piece of paper fold through the third dimension, one would see the shadow of the fold. In the process of folding, one would see $\omega_{2}$ become deformed as it nears the light source and eventually flip across $\partial \Omega_{I}$ to become $-\omega_{2}$ [figure 3a]. In other words, when folding, a side crosses over the common boundary and concludes upside-down. The same basic concept is true when folding a three dimensional figure. However, instead of $\partial \Omega_{I}$ being a line, it is a face, and, in contrast with concluding upside-down, it terminates in an inside-out position [figure $3 \mathrm{~b}$ ]. Being able to visualize higher dimensional folding rules is key to an understanding of a transplantation proof in 3-space.

\section{ISOSPECTRAL DOMAINS IN EUCLIDEAN 3-SPACE}

A number of isospectral regions have been presented in Euclidean and hyperbolic 2spaces. These have utilized what is known as a transplantation proof of isospectrality, which can be done through folding and translating. We now present a pair of our three dimensional isospectral domains [figure 4] and describe the transplantation of the eigenfunctions betwixt these regions. a. Transplantation of the eigenfunctions in 3-space

When dealing with a wave equation of the form in this paper, the concepts of linear superposition can be highly beneficial. This concept simply states that particular solutions of a linear differential equation can be summed to obtain the general solution. In the case of the regions here, consider the domain, $\Omega$, to be divided up into $m$ subregions, $\omega_{i}$, such that

$$
\Omega=\bigcup_{i=1}^{m} \omega_{i}
$$

If this is the case, we can assign a particular solution to a given subregion, so that $\psi_{i}$ is defined in $\omega_{i}$. Therefore the full solution is given by

$$
\varphi=\sum_{i=1}^{m} \psi_{i}
$$

In order to simplify notation, we shall denote $\psi_{1}$ as $A, \psi_{2}$ as $B$, and so on. In the case of the regions presented in figure 4 , it is convenient to divide $\Omega_{1}$ into seven tetrahedral subdomains, such that

$$
\varphi=\sum_{i=1}^{7} \psi_{i}=A+B+C+D+E+F+G
$$




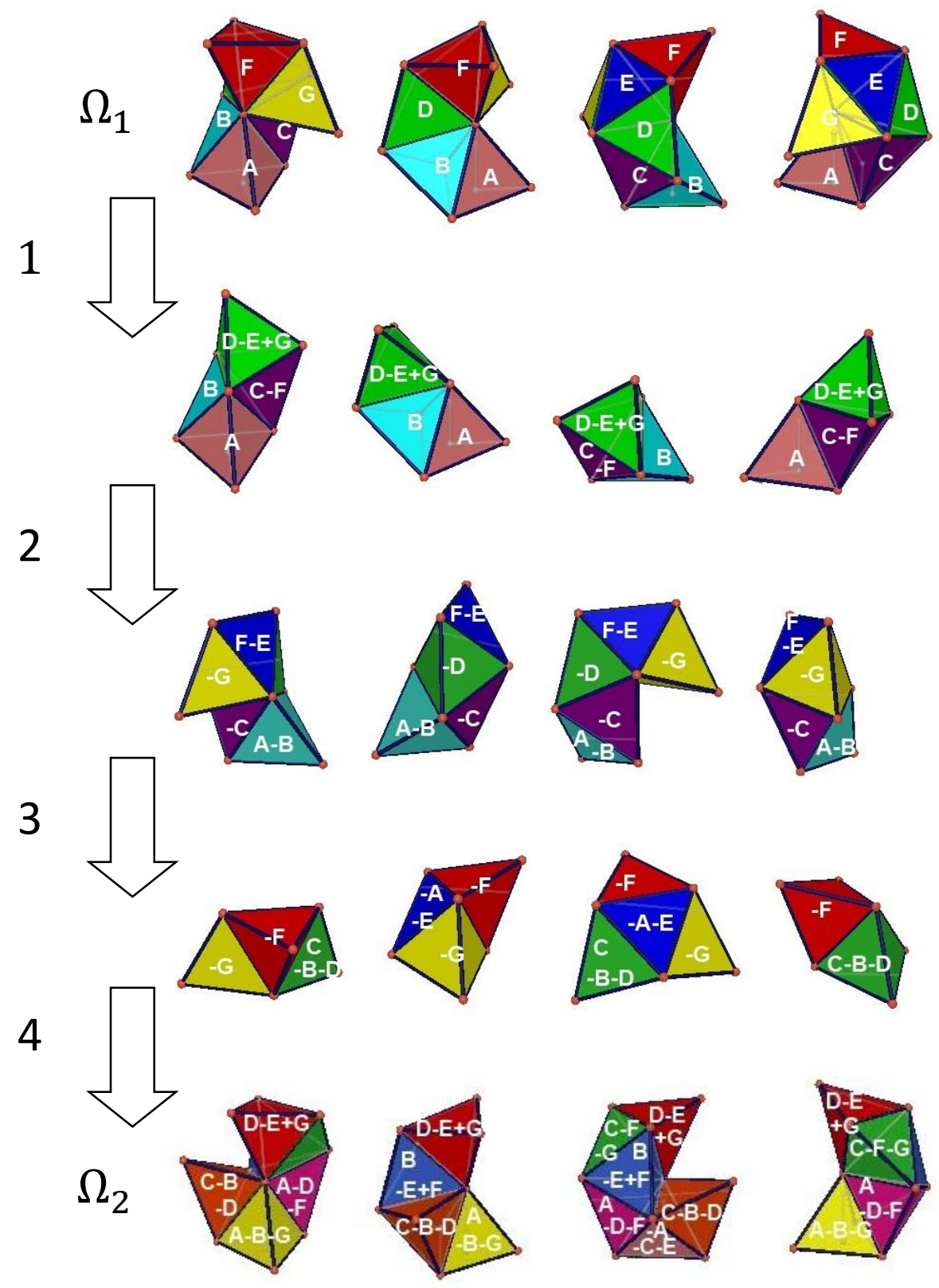

Figure 5. Methodology for transplanting $\varphi_{1}$ onto $\varphi_{2}$. Once again, note that the colors are simply to aid in the visualization of the domains and have no other significance.

By utilizing the assertions presented in section 2.2, we can now form a map to transplant the eigenfunctions of $\Omega_{1}$ to those of $\Omega_{2}$. By combining section 2.2 with the concepts of linear superposition, we can infer that if $A$ is folded onto $B$, the particular solution on the folded region would be $(B-A)$. If this, in turn, were to be folded onto $C$, the superposed eigenfunction would be $(C-B+A)$. Figure 5 presents the folding methods to accomplish mapping $\varphi_{1}$ onto $\varphi_{2}$.

The folding process for showing $\Omega_{1}$ and $\Omega_{2}$ to be isospectral, in the case of our given domains, consists of four basic steps although there may be greater numbers for more complicated regions [figure 5]. 

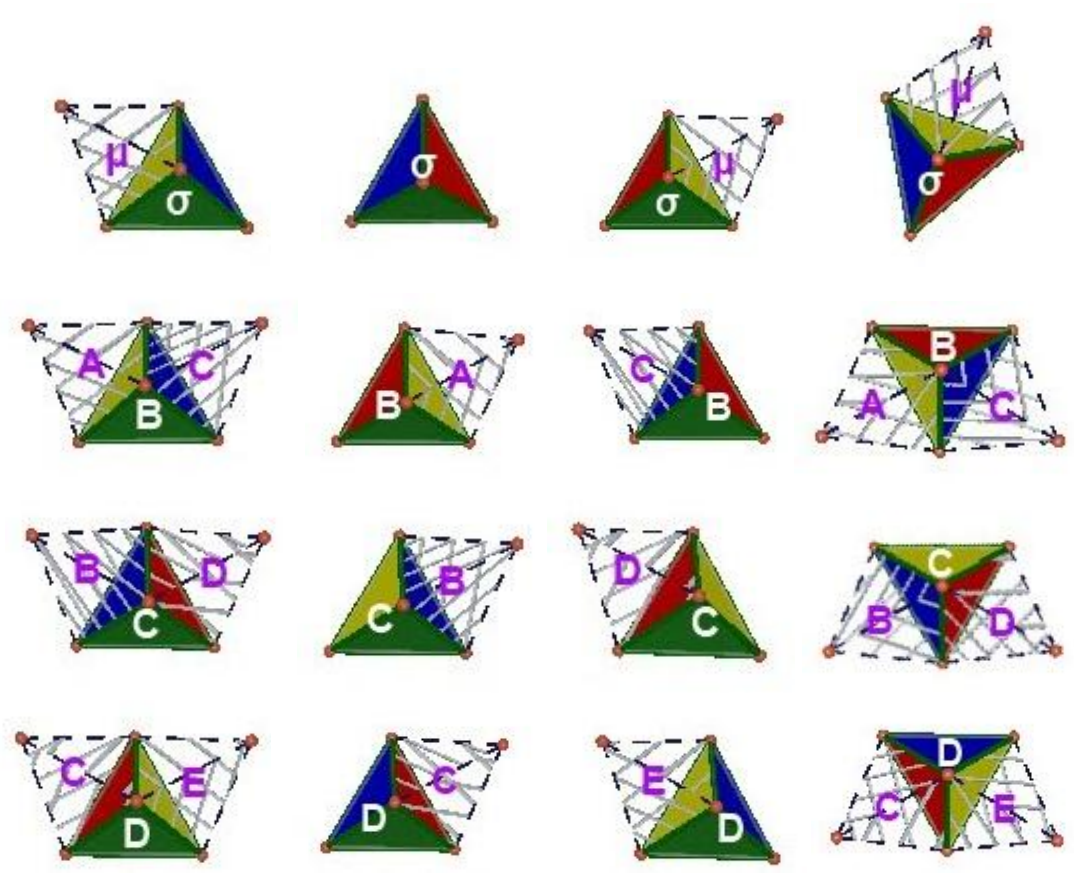

Figure 6. The subregion $\sigma$, from $\Omega_{2}$, and the subregions $B, C$, and $D$, from $\Omega_{1}$, are shown. The colorations of the faces denote the similar boundaries between the subregions due to the principle of reflection, and the wireframes show the connectivity in the full domain.

Step 1: Starting with $\Omega_{1}$, fold $G$ onto $E$, then fold both $(E-G)$ onto $D$, thereby creating the superpositions of $(D-E+G)$ and $(C-F)$.

Step 2: Starting with $\Omega_{1}$, turn it 'inside out,' thereby obtaining $-\Omega_{1}$. Fold $-F$ onto $-E$, then fold $-A$ onto $-B$. This creates the superpositions of $(A-B)$ and $(F-E)$.

Step 3: Again, start with $-\Omega_{1}$ and fold $-C$ onto $-D$, thereby creating the superpositions of $(-A-E)$ and $(C-B-D)$.

Step 4: By combining the figures created in steps 1,2, and 3 in such a way that each tetrahedral subregion becomes a superposition of three eigenfunctions from $\Omega_{1}, \quad \Omega_{2}$ is obtained.

Once this map has been created, three conditions must be satisfied. The map must be nonsingular, the Dirichlet boundary conditions must be upheld, and the eigenfunctions must have a continuous derivative within the region. The first condition is easily satisfied by showing that the map can be inversed so that it maps $\varphi_{2}$ onto $\varphi_{1}$. This is proven in a straightforward manner by either using the same methods in reverse or by creating a transplantation matrix, $M$, to encode the folding rules and show that $\operatorname{det}(M) \neq 0$.

The second and third conditions are more challenging to verify due to the difficulty in visualizing $\partial \Omega$ and $\partial \Omega_{I}$ in three dimensions. Condition two states that the function must vanish on $\partial \Omega$. Although the eigenfunctions and eigenvalues cannot be analytically computed, they can be equated between domains. As shown in figure 5, every eigenfunction in a given tetrahedral subdomain of $\Omega_{1}$ is a superposition of three eigenfunctions from $\Omega_{1}$. The process of assuring that $\left.\varphi\right|_{\partial \Omega}=0$ consists of simply checking that the eigenfunction superpositions go to zero at every boundary. With domains in 3-space, this can become challenging due to the difficulty in visualization. As an example, we take a test subregion from $\Omega_{2}$ such as the tetrahedron containing $(C-B-D)$, which shall be denoted as $\sigma$ for simplicity. In order to test the boundary conditions, we must refer back 

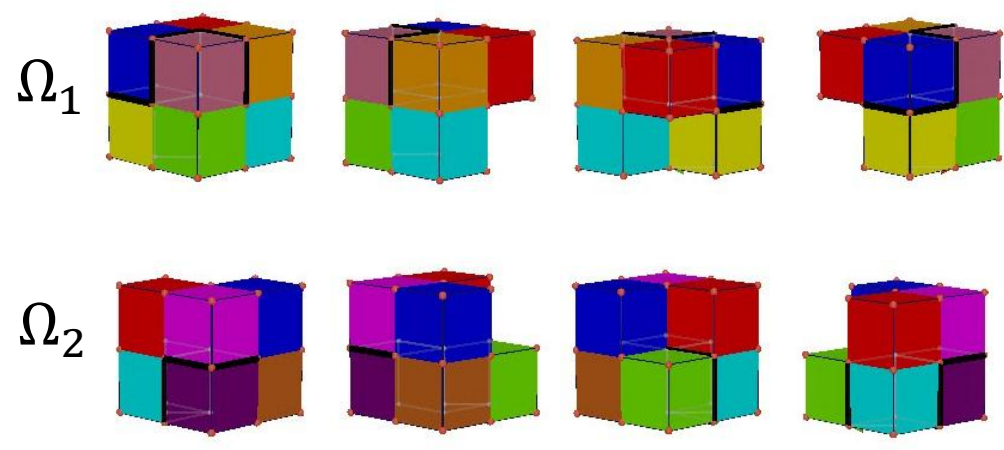

Figure 7a. These two isospectral domains were created from the base of a hexahedron. The thick black lines visible on some of the boundaries of the subregions are to show detachment, i.e. the two subregions on either side are disjoint. The colors have no other significance beyond aiding in visualization.
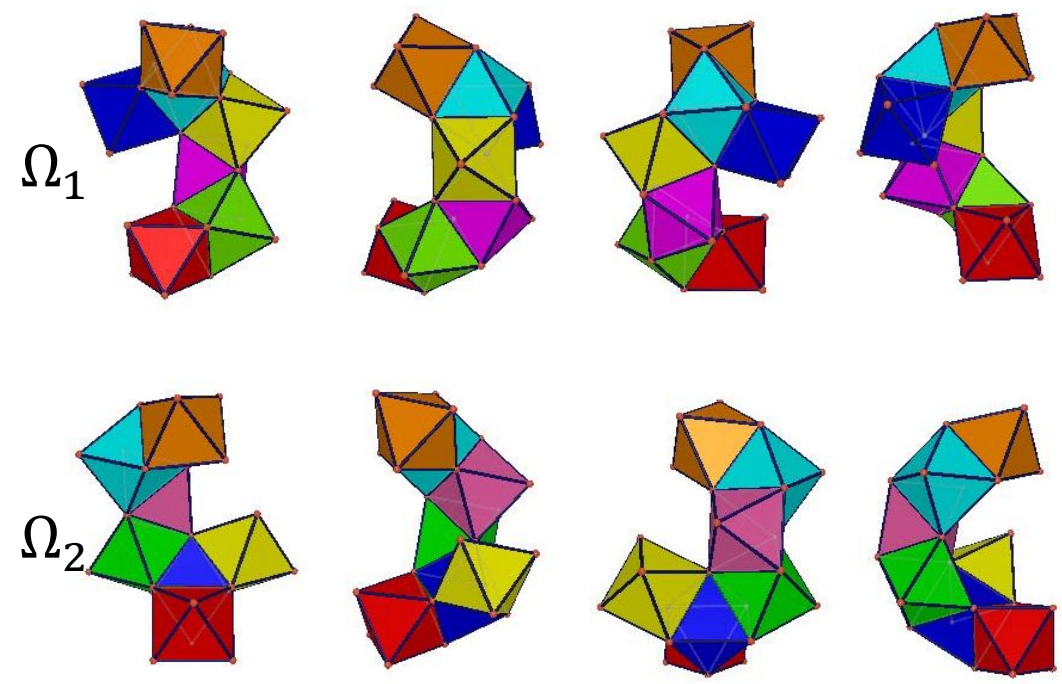

Figure 7b. This pair of isospectral domains was created from an octahedral base. Once again, the colorations are simply for the eye.

to $\Omega_{1}$ and equate the eigenfunctions [figure $6]$.

First we look at the blue edge of $\sigma$ and compare it to that of $C, B$, and $D$. Upon investigation, we see that $\left.D\right|_{\text {blue }}=0$ because it is an exterior boundary, and that, due to their sharing of the common face, $\left.C\right|_{\text {blue }}=\left.B\right|_{\text {blue }} ;$ thus $\left.\sigma\right|_{\text {blue }}=(C-B-$ $D)\left.\right|_{\text {blue }}=\psi-\psi-0=0$, where $\psi$ is an arbitrary function. Now turning attention to the red edge, we find that $\left.B\right|_{\text {red }}=0$ and $\left.C\right|_{\text {red }}=\left.D\right|_{\text {red }}$. This being the case, $\left.\sigma\right|_{\text {red }}=$ 0 . Evaluation on the green edge yields $\left.C\right|_{\text {green }}=\left.B\right|_{\text {green }}=\left.D\right|_{\text {green }}=0, \quad$ thereby making $\left.\sigma\right|_{\text {green }}=0$. By carrying out this process for every face on $\partial \Omega_{2}$, the Dirichlet boundary conditions will be proven upheld. Condition three is proven in a similar manner by assuring that $\varphi_{2}$ continuous derivative across all $\partial \Omega_{I}$. By again taking the subregion of $\sigma$, we look at the yellow interior face. In order to assure the continuous derivative, we must also consider the subregion connected by the yellow face, $(-A-E-C)$, denoted as $\mu$. By looking at $\Omega_{1}$, we see that $\left.B\right|_{\text {yellow }}=\left.A\right|_{\text {yellow }}$; $\left.D\right|_{\text {yellow }}=\left.E\right|_{\text {yellow }} ;$ and $\left.C\right|_{\text {yellow }}=0$. By this 
account, $\left.\sigma\right|_{\text {yellow }}=\left.\mu\right|_{\text {yellow }}$. Through this method of equating, we have shown that the derivative is continuous across this interior face. Once again, by continuing this process for all $\partial \Omega_{I}, \varphi_{2}$ is shown to have a continuous derivative throughout $\Omega_{2}$.

\section{b. Other isospectral domains in 3-space}

Through the means presented in section 3.1, we present a number of other isospectral domains in Euclidean 3-space. To create a pair of three dimensional isospectral domains can be done simply by adding a unit of depth to a preexisting two dimensional pair. This simple extension, however, does not create interesting results. The domains presented here are created by using the platonic solids as a base subregion [figure $7 \mathrm{a} \&$ 7b]. Only two domains are presented in the paper in order to portray the concept. Additional similar regions can be obtained by taking simple mutations of the base subregion.

\section{Concluding remarks}

Applications of these results mainly lie in that of particle and quantum physics. By altering the Dirichlet boundary conditions to suit the Schrödinger equation, isospectral potential energy systems of particles may be able to be produced and studied.

Further research includes the extension of transplantation into higher dimensions. In the near future, we shall be working on this question in 4-space by using these same basic methods. One of the largest questions still remaining in this field is whether or not there exist pairs of convex isospectral domains in given spaces. Convex isospectral domains have been shown to exist in $\mathbb{H}^{2}$ and $\mathbb{R}^{n}$, for $n \geq 4$ [see $3 \& 4$, but none have been proven, nor disproven, to exist in $\mathbb{R}^{2}$ or $\mathbb{R}^{3}$.

\section{ACKNOWLEDGEMENTS}

I would like to thank Dr. Michael Roth, of the University of Northern lowa, for supporting me in this endeavor and for teaching me the basics of partial differential equations, eigenvalues problems, and boundary conditions. I would also like to thank Dr. Jason Grout, of Drake University, for introducing me to the Sage network and helping to create the visuals used in my preliminaries. Finally, I would like to give credit to Dr. David Kung, of St. Mary's College of Maryland, for bringing this topic of isospectrality to my attention through a lecture [see 7].

\section{REFERENCES}

1. Bérard, Pierre. "Transplantation et isospectralité I." Mathematische Annalen 292, no. 3 (1992): 547-559.

2. Buser, Peter; Conway, John; Doyle, Peter; and Semmler, Klaus-Dieter. "Some planar isospectral domains." International Mathematics Research Notices 25, no. 3 (1994): 225-246.

3. Gordon, Carolyn; and Webb, David. "Isospectral convex domains in Euclidean space." Mathematical Research Letters 1, no. 5 (1994): 539545.

4. Gordon, Carolyn; and Webb, David. "Isospectral convex domains in the hyperbolic plane." Proceedings of the American Mathematical Society 120, no. 3 (1994): 981-983.

5. Gordon, Carolyn; Webb, David; and Wolpert, Scott. "One cannot hear the shape of a drum." Bulletin of the American Mathematical Society 27, no. 1 (1992): 134-138.

6. Kac, Mark. "Can one hear the shape of a drum?" The American Mathematical Monthly 73, no. 4 (1966): 1-23.

7. Kung, David. "How math made modern music mad irrational!" Lecture, Brigham Young University, Provo, UT, March 18, 2011. 


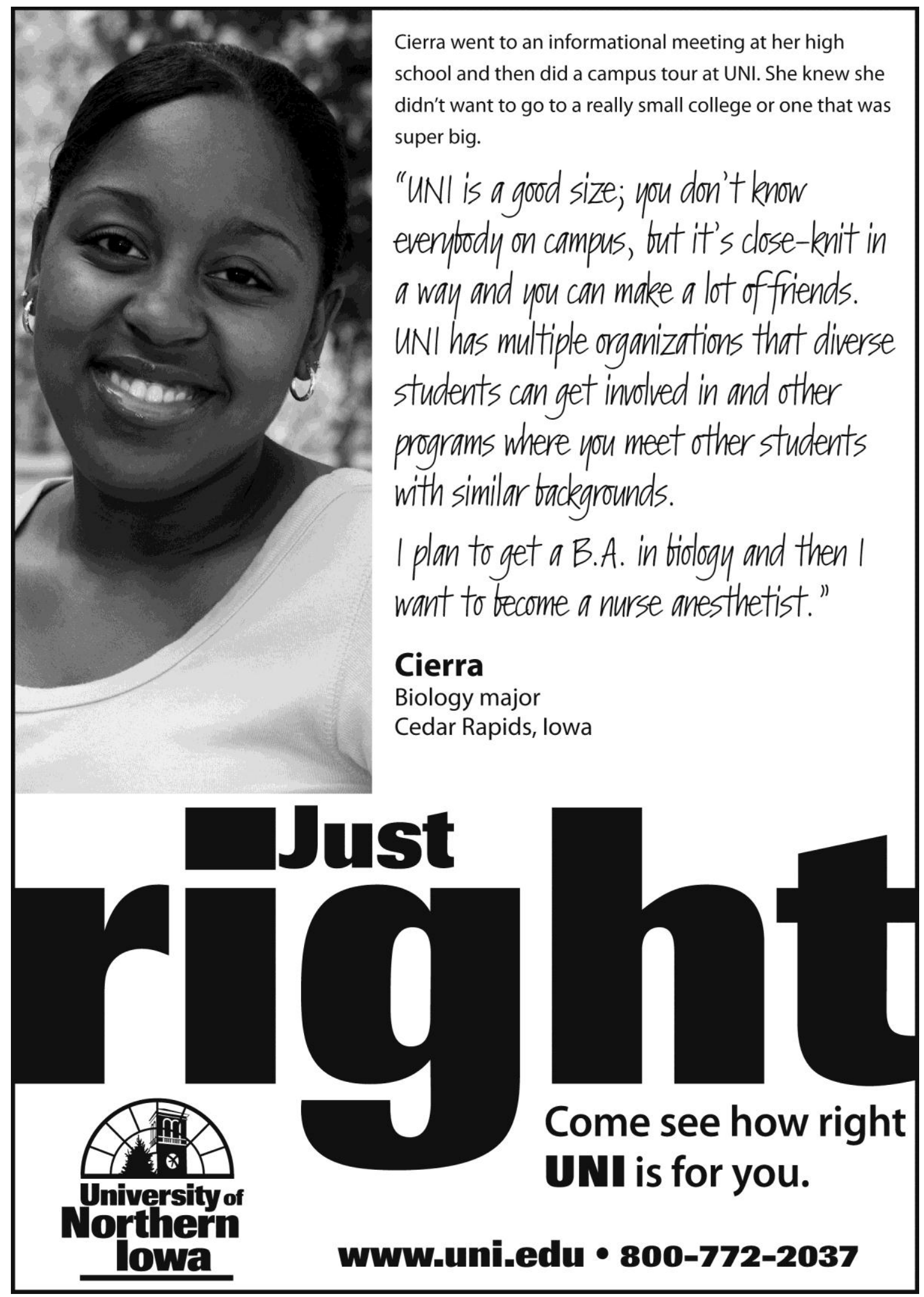

